\title{
Erythema chronicum migrans and salmon fishing in Alaska: an enigma resolved by microbiology laboratory
}

\author{
Silvio Daffara', Valeria Brazzelli', Giorgia Ronzi², Nadia Vicari', Daniela Barbarini', Dario Pistone', \\ Massimo Pajoro ${ }^{3}$, Massimo Fabbi ${ }^{3}$, Claudio Bandi ${ }^{4}$, Piero Marone' \\ I SC di Virologia e Microbiologia, Fondazione IRCCS San Matteo, Pavia \\ 2 Clinica Dermatologica, Fondazione IRCCS San Matteo, Pavia \\ 3 Istituto Zooprofilattico della Lombardia ed Emilia, Sezione di Pavia \\ 4 Laboratorio di Parassitologia molecolare, Università di Milano
}

Key words: Erythema chronicum migrans, Borreliosis, Borrelia afzelii

Eritema cronico e pesca di salmoni in Alaska: un enigma risolto dal laboratorio di Microbiologia

\section{SUMMARY}

A 50-year-old man, pentathlete and fond of salmon fishing, after returning from Alaska was referred to our Division for an erythematous and indolent lesion on the right thigh gradually enlarging from the right knee to the right rear and the buttock. The patient was clinically diagnosed with figurate erythema. Laboratory analysis demonstrated a moderate leukocytosis and hypergammaglobulinemia, accompanied by serological markers of past contact with noted EBV and CMV.A "punch" biopsy of the edge of the lesion showed a superficial and deep perivascular and interstitial infiltrate of lymphocytes, eosinophiles and a few plasma cells, consistent with a borrelial infection. Lyme serology (IFI) suggested a questionable borderline positivity; subsequent investigations by ELISA and Western Blot were both positive, leading to the diagnosis of erythema chronicum migrans in course of borreliosis. The diagnosis was further confirmed by positive PCR for Borrelia burgdorferi sensu lato. The erythema resolved after a 2-week doxycycline treatment $(2 \times 100 \mathrm{mg}$ die). The amplification product (I6S rDNA gene) obtained from skin biopsy was sequenced using standard $A B I$ technology, and confirmed the identification of a member of $B$. burgdorferi sensu lato (sl) complex. Since this sequence was not useful to identify the genospecies, further studies were conducted employing a nested PCR targeted on the internal transcribed spacer (ITS) of B. burgdorferi, following a protocol previously described (I). The ITS sequence showed $100 \%$ identity with $B$. afzelii, a species not reported from North America, thus prompting us to conclude that the patient was not infected by $B$. afzelii during the fishing trip in Alaska.

La borreliosi o malattia di Lyme è una patologia a carattere multisistemico causata da spirochete del genere Borrelia o appartenenti a $B$. burgdorferi sensu lato (sl) complex, trasmessa dalla puntura di zecche del genere Ixodes (4). L’eritema cronico migrante (ECM) è di solito la manifestazione più precoce di alcune forme di borreliosi; esso è costituito da una lesione maculo-papulosa di colorito rossastro di circa $10-20 \mathrm{~cm}$ che va espandendosi con la zona centrale che tende ad assumere un colorito più chiaro con l'evolvere della lesione (5). Caratteristicamente asintomatica, può peraltro associarsi a sensazione di calore, prurito, dolore, iperestesia o disestesia (2). La zona colpita appare più calda della cute circostante. In un 50\% dei soggetti l'ECM può accompagnarsi a malessere, astenia, febbre, linfoadenomegalia regionale. Se non trattato, l'ECM perdura per settimane o mesi ed è talvolta seguito dalla comparsa di un rush fugace. Il decorso viene bruscamente modificato da una appropriata terapia antibiotica, che impedisce il passaggio alla forma secondaria disseminata.

In particolare l'eritema cronico migrante fa parte di un gruppo di dermatosi, noto come eritema figurato, insieme all'eritema anulare centrifugo, all'eritema girato repens, all'eruzione fissa da medicamenti e all'eritema multiforme.

Un uomo di 50 anni, pentatleta e appassionato di pesca al salmone, dopo essere tornato dall'Alaska, nell'agosto 2008 giunge all'Ambulatorio della Divisione di Dermatologia della Fondazione IRCCS Policlinico S. Matteo di Pavia a seguito della comparsa di una lesione eritematosa e indolente alla coscia destra, progressivamente estesa al ginocchio destro e alla radice dell'arto inferiore destro posteriormente fino alla regione glutea con progressivo impallidimento della zone centrale della lesione stessa (Figura I). Viene posta diagnosi clinica di eritema figurato e pertanto vengono eseguiti accertamenti ematochimici, sierologici e viene effettuata biopsia cutanea per esame istologico.

Gli esami di laboratorio mostrano modesta leucocitosi e iper-

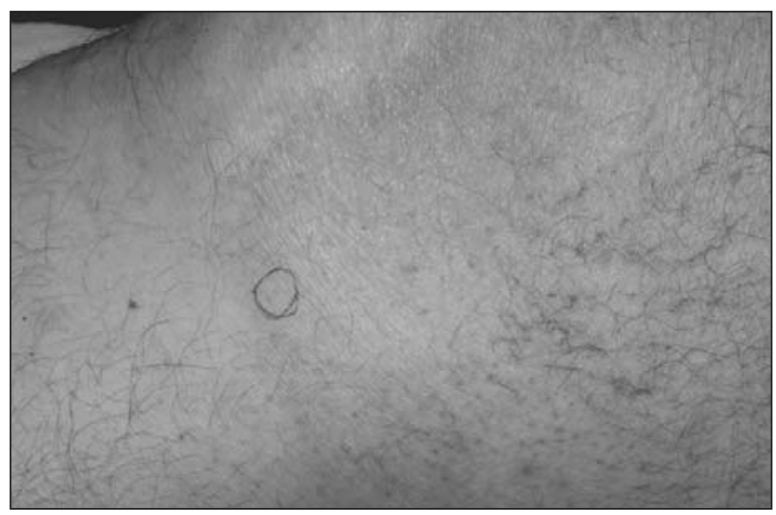

Figura I. Eritema cronico migrante

gammaglobulinemia, mentre le sierologie virali evidenziano pregresso contatto con EBV e CMV. Viene eseguita biopsia cutanea tipo "punch" dal bordo della lesione il cui esame istologico mette in evidenza la presenza di un infiltrato superficiale e profondo perivascolare e interstiziale composto da linfociti, qualche eosinofilo e alcune plasmacellule. L'aspetto clinico e l'aspetto istologico caratterizzato soprattutto dalla presenza di plasmacellule suggeriscono di eseguire la sierologia per la ricerca di anticorpi anti-B. burgdorferi (IFI), che dimostra una dubbia positività borderline. Indagini successive con metodiche ELISA e Western Blot risultano entrambe positive e consentono di porre diagnosi di eritema cronico migrante in corso di borreliosi. La diagnosi è ulteriormente confermata mediante positività in PCR per B. burgdorferi sl (Figura II). Quest'ultima è stata realizzata su DNA estratto da biopsia cutanea della lesione, utilizzando una coppia di primer, BBLD5' e BBLD3', in grado di amplificare il gene codificante per l'rRNA 16S, secondo il protocollo descritto da

\section{Corresponding author: Silvio Daffara}

SC di Virologia e Microbiologia - Fondazione IRCCS San Matteo

27100 Pavia - Via Taramelli 5 - Tel.: 0382502702 - Fax: 0382502282

E-mail: silviodaffara@yahoo.it 
Marconi e Garon (3).

Il paziente è stato trattato con doxiclina $100 \mathrm{mg} 2$ volte/die per due settimane; la terapia ha determinato la risoluzione del quadro con scomparsa della lesione cutanea.

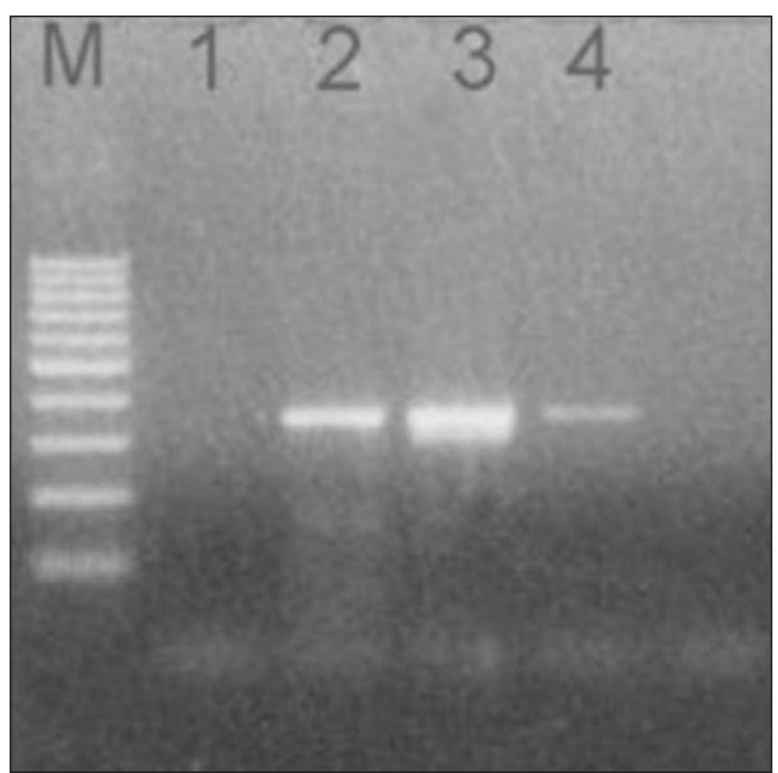

Figura II. Elettroforesi in gel d'agarosio della PCR per Borrelia burgdorferi sensu lato.

\begin{tabular}{ccl}
\hline$M$ & $=$ & 100 bp ladder \\
\hline I & $=$ & controllo negativo \\
\hline 2 & $=$ & biopsia cutanea (357bp) \\
\hline 3 & $=$ & C + B. afzelii TQ \\
\hline 4 & $=$ & C + B. afzelii diluito $1: 10$ \\
\hline
\end{tabular}

Il prodotto di amplificazione (16S rDNA) ottenuto dalla biopsia cutanea è stato sequenziato con tecnologia $\mathrm{ABI}$, per confermare il coinvolgimento di spirochete appartenenti a $B$. burgdorferi sl complex. Poiché la sequenza non permette di identificare la genospecie, sono state effettuate ulteriori analisi mediante nested-PCR della regione ITS (internal transcribed spacer-spaziatore ribosomale) di $B$. burgdorferi secondo un protocollo descritto in precedenza. La sequenza ITS ottenuta presenta un'identità del 100\% con B. afzelii. Poiché questa specie non è descritta in Nord America, si può ipotizzare che il paziente non sia stato infettato da B. afzelii dalla puntura della zecca durante il viaggio in Alaska.

Questo caso è particolarmente significativo poiché sono presenti tutte le caratteristiche cliniche e microbiologiche necessarie per una corretta diagnosi ed è stato studiato attraverso numerose indagini laboratoristiche, dalla sierologia all'istologia e infine con la biologia molecolare. In particolare, la sequenza della regione ITS ha consentito di identificare il microrganismo coinvolto in questa infezione come B. afzelii. La ricerca di CB è stata finanziata da MIUR COFIN e ISPESL.

\section{BIBLIOGRAFIA}

1. Chu CY, Jiang BG, Zhao QM, Wu XM. Presence of pathogenic Borrelia burgdorferi sensu lato in ticks and rodents in Zhejiang, south.east China. J Med Microbiol 2008; 57(8): 980-5.

2. Lipsker D. Dermatological aspect of Lyme borreliosis Med. Mal Infect 2007; 37: 540-7.

3. Marconi RT, Lubke L, Hauglum W, Garon CF. Species-specific identification of and distinction between Borrelia burgdorferi genomic groups by using 16S rRNA-directed oligonucleotide probes. J Clin Microbiol 1992; 30(3): 628-32.

4. Steree AC. Lyme disease N. Eng J Mol 1989; 321: 586-96.

5. Strle F, Nelson JA, Ru' ìc Sablijic E, et al. European Lyme borreliosis. 231 culture-confirmed cases involving patient with erythema migrans. Clin Inf Dis 1996; 23: 61-5. 